OPEN ACCESS

Edited by:

S. Venkata Mohan, CSIR-Indian Institute of Chemical

Technology, India

Reviewed by:

Yu-Shen Cheng,

National Yunlin University of Science and Technology, Taiwan

G. Venkata Subhash,

Reliance Industries, India

*Correspondence:

Calvin A. Henard

calvin.henard@nrel.gov

Specialty section:

This article was submitted to

Bioenergy and Biofuels,

a section of the journal

Frontiers in Bioengineering and

Biotechnology

Received: 04 November 2016

Accepted: 27 December 2016

Published: 17 January 2017

Citation:

Henard CA, Guarnieri MT and Knoshaug EP (2017) The

Chlorella vulgaris S-Nitrosoproteome under Nitrogen-Replete and-Deplete Conditions.

Front. Bioeng. Biotechnol. 4:100. doi: 10.3389/fbioe.2016.00100

\section{The Chlorella vulgaris $S$-Nitrosoproteome under Nitrogen-Replete and -Deplete Conditions}

\author{
Calvin A. Henard*, Michael T. Guarnieri and Eric P. Knoshaug \\ National Bioenergy Center, National Renewable Energy Laboratory, Golden, CO, USA
}

Keywords: Chlorella, biofuels, microalgae, S-nitrosylation, nitric oxide

Oleaginous microalgae synthesize and accumulate large quantities of lipids that are promising feedstocks for the production of biofuels (Hu et al., 2008; Williams and Laurens, 2010; Day et al., 2012; Quinn and Davis, 2015). The algal species Chlorella vulgaris accumulates triacylglycerides that dominate its cellular composition ( $>60 \%$ lipid based on dry cell weight) when cultured in medium lacking a nitrogen source (Guarnieri et al., 2011; Ikaran et al., 2015), which is a "lipid trigger" in an array of microalgae. As such, C. vulgaris represents a model algal species for examination of lipid accumulation mechanisms and a potential deployment organism in industrial algal biofuels applications. C. vulgaris has been extensively characterized biochemically and physiologically (Converti et al., 2009; Liang et al., 2009), and de novo-generated transcriptomic and proteomic datasets have indicated that post-transcriptional and -translational mechanisms likely govern lipid accumulation in response to nitrogen starvation (Guarnieri et al., 2011, 2013). However, the specific mechanisms underlying lipid biosynthesis in response to nitrogen stress remain elusive.

Nitric oxide (NO) has received much attention as a signaling molecule due to its ability to react specifically with a limited number of biomolecules, primarily mediating physiological changes by modifying proteins in diverse domains of life, including plants, animals, and prokaryotes. This diatomic radical targets $[\mathrm{Fe}-\mathrm{S}]$ clusters of dehydratases in several central metabolic pathways and can react with redox active sulfhydryls in cysteines to produce $S$-nitrosothiols. Protein $S$-nitrosylation by NO has been shown to play important roles in an array of cellular responses in organisms ranging from prokaryotes to metazoans. Previous studies have identified several proteins as targets of NO in higher plants, confirming S-nitrosylation as a key post-translational mechanism governing cell signaling in these organisms (Zaffagnini et al., 2016). Additional studies in the green microalga Chlamydomonas reinhardtii have identified hundreds of $S$-nitrosylated proteins after treatment with S-nitrosoglutathione (Samuel et al., 2014), supporting S-nitrosylation as a widespread posttranslational mechanism employed by autotrophic organisms.

Based on the nitrate reductase-dependent NO production observed in higher plants and microalgae (Sakihama et al., 2002; Mur et al., 2013), we evaluated whether NO is produced by C. vulgaris when cultured in medium with nitrate as the sole nitrogen source. Using the NO-reactive fluorophore 4,5-diaminofluorescein, we detected NO produced by $C$ vulgaris during logarithmic growth in modified Bold's Basal Medium (BBM) supplemented with $3 \mathrm{mM}$ sodium nitrate (Figure 1). The NO detected in algae exposed to nitrite in acidified BMM ( $\mathrm{pH} 4.5$ ), a condition that non-enzymatically generates high concentrations of NO congeners (Henard et al., 2014), was 10-fold higher than the concentration endogenously produced by this organism under nitrogen-replete growth (Figure 1A). NO was detected throughout the cell cytoplasm of cells grown in the presence of acidified nitrite, with more intense staining observed in the chloroplast (Figure 1B). 

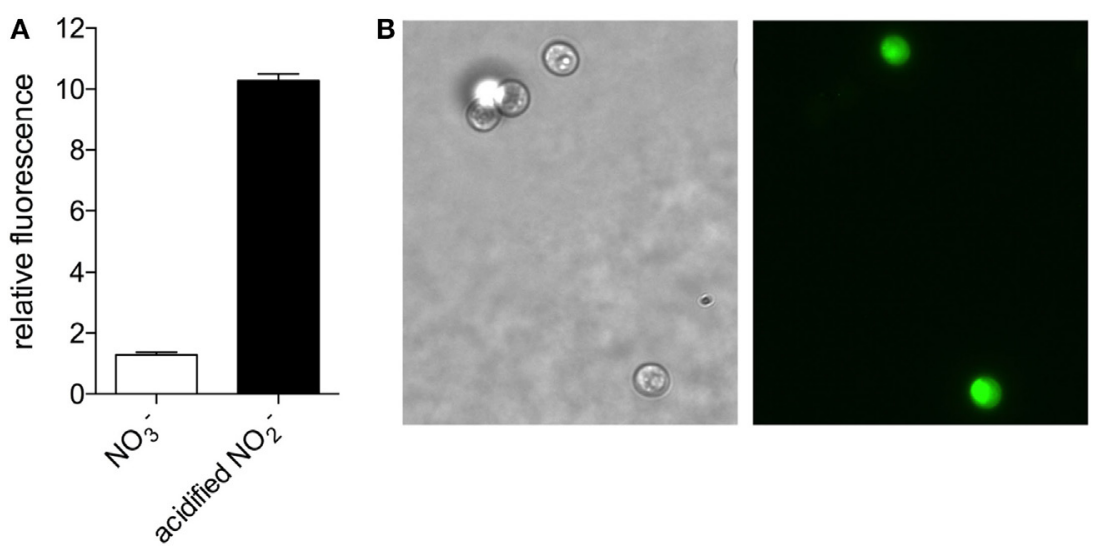

FIGURE 1 | Nitric oxide production in Chlorella vulgaris detected by 4,5-diaminofluorescein staining. (A) C. vulgaris grown in Bold's Basal Medium (BBM) with $3 \mathrm{mM} \mathrm{NaNO}_{3}$ (white bar) or treated with $1 \mathrm{mM} \mathrm{NaNO}_{2}$ in $\mathrm{BBM} \mathrm{pH} 4.5$ for $1 \mathrm{~h}$ (black bar) were stained with 4,5-diaminofluorescein to detect nitric oxide production fluorometrically. (B) Bright field (left panel) and fluorescence (right panel) microscopy of a representative $\mathrm{NaNO}_{2}$-treated sample.

To assess whether the nitrate-derived NO is biologically active, we determined the $S$-nitrosoproteome of C. vulgaris in the presence or absence of nitrate. The biotin switch method identified 40 proteins that are endogenously $S$-nitrosylated in C. vulgaris during active growth (Table 1, nitrogen replete). $S$-nitrosylated proteins were identified from a variety of metabolic pathways including photosynthesis and light capture, the Calvin-Benson cycle and carbon capture, protein synthesis, the TCA cycle, stress response, fatty acid biosynthesis, and cell structure. Several of the $S$-nitrosylated proteins identified here have confirmed orthologous targets of NO in diverse organisms, including plants and algae (Zaffagnini et al., 2016). Interestingly, $80 \%$ of the $S$-nitrosothiols detected during active growth were undetected under nitrogen starvation (Table 1, nitrogen deplete) with only 9 of the 40 proteins identified as being $S$-nitrosylated during nitrogen-replete conditions still identified during nitrogen starvation conditions. Collectively, the decrease in $S$-nitrosylated proteins identified under nitrogen starvation suggests S-nitrosylation occurs primarily during growth in a nitrogen-replete environment, presumably due to nitrate reductase-mediated NO production. Several proteins identified here have been previously identified as $S$-nitrosylated in plants, mammals, and prokaryotes in the presence of exogenous NO donors; however, our data represent targets $S$-nitrosylated by endogenously produced NO via nitrate metabolism.

S-nitrosylation of proteins identified here could have direct roles in lipid accumulation in C. vulgaris. The differential S-nitrosylation of biotin carboxylase observed during nitrogen-deplete and -replete conditions may play a direct role in C. vulgaris lipid accumulation. Biotin carboxylase is a subunit of the acetyl-CoA carboxylase (ACCase) complex and production of malonyl-CoA by this enzyme complex is considered the first committing step in lipid triacylglycerol (TAG) biosynthesis. We previously noted that this enzyme is upregulated during nitrogen starvation (Guarnieri et al., 2013), but, interestingly, we only detected $S$-nitrosylated biotin carboxylase during growth in the presence of nitrate. We should note that since biotin is a cofactor utilized by this enzyme, it is possible that even in the absence of being $S$-nitrosylated, it can potentially bind to the streptavidin matrix and be enriched with $S$-nitrosothiols during the biotin switch assay. However, although biotin carboxylase is upregulated during nitrogen starvation, we did not detect this enzyme under nitrogen-deplete conditions, indicating that its enrichment in nitrogen-replete samples was not simply biotinylated enzyme. Given the low level of neutral lipids produced during nitrogenreplete growth, biotin carboxylase represents a potential proteinengineering target to improve TAG accumulation during active growth.

Dihydrolipoyl dehydrogenase (DHLD) functions as a subunit in the $\alpha$-ketoacid dehydrogenase complexes. As a subunit in the pyruvate dehydrogenase complex, DHLD is involved in converting pyruvate to acetyl-CoA and has been implemented in increasing lipid production by providing additional lipid precursors (acetyl-CoA) in Chlorella protothecoides grown heterotrophically on sugarcane bagasse hydrolyzate (Mu et al., 2015). DHLD has also been shown to be $S$-nitrosylated in plants responding to pathogens, cold, and abiotic stress (Maldonado-Alconada et al., 2011; Ortega-Galisteo et al., 2012; Puyaubert et al., 2014). As with biotin carboxylase, DHLD is only $S$-nitrosylated during nitrogenreplete growth and represents another potential target for protein engineering to eliminate the negative effects of S-nitrosylation on this enzyme.

Six enzymes involved in the Calvin cycle were identified as $S$-nitrosylated including ribulose bisphosphate carboxylase/oxygenase, phosphoglycerate kinase, three isozymes of glyceraldehyde-3-phosphate (GAPDH), and fructose bisphoshate aldolase (FBA). GAPDH has been previously reported to be $S$-nitrosylated in plants responding to cold, ozone, high light, salt, or pathogen-induced stress (Maldonado-Alconada et al., 2011; Astier and Lindermayr, 2012; Puyaubert et al., 2014; Vanzo et al., 2014). GAPDH regulation in response to nitrogen stress in C. vulgaris appears to be quite complex as 
TABLE 1 | Chlorella vulgaris S-nitrosylated proteins under nitrate-replete and -deplete conditions.

\begin{tabular}{|c|c|c|c|c|c|c|c|c|}
\hline \multirow[b]{2}{*}{$\begin{array}{l}\text { Scaffold. } \\
\text { gene }\end{array}$} & \multirow[b]{2}{*}{ BLASTP match } & \multirow[b]{2}{*}{ e Value } & \multirow[b]{2}{*}{$\begin{array}{c}\text { Molecular } \\
\text { weight (kDa) }\end{array}$} & \multicolumn{2}{|c|}{ Replete } & \multicolumn{2}{|c|}{ Deplete } & \multirow[b]{2}{*}{$\begin{array}{c}\text { \# cysteine } \\
\text { residues }\end{array}$} \\
\hline & & & & $\begin{array}{l}\text { \# unique } \\
\text { peptides }\end{array}$ & $\begin{array}{c}\% \\
\text { coverage }\end{array}$ & $\begin{array}{l}\text { \# unique } \\
\text { peptides }\end{array}$ & $\begin{array}{c}\% \\
\text { coverage }\end{array}$ & \\
\hline \multicolumn{9}{|c|}{ Photosynthesis } \\
\hline 291.25 & Photosystem I subunit VII & $3.80 \mathrm{E}-52$ & 9 & 3 & 38 & & & 9 \\
\hline 432.15 & Chlorophyll a/b-binding protein & 1.00E-152 & 27 & 4 & 42 & & & 2 \\
\hline 772.94 & Chloroplast light-harvesting complex II protein precursor & $0.00 \mathrm{E}+00$ & 52 & 7 & 28 & 4 & 8 & 2 \\
\hline 1102.1 & Photosystem I p700 chlorophyll a apoprotein a1 & $0.00 \mathrm{E}+00$ & 83 & 3 & 3 & 2 & 2 & 4 \\
\hline 1102.11 & Photosystem I p700 apoprotein a2 & $0.00 \mathrm{E}+00$ & 70 & 3 & 5 & & & 3 \\
\hline 1102.7 & Photosystem II 47 kDa protein & $0.00 E+00$ & 56 & 5 & 8 & & & 3 \\
\hline 1280.117 & Photosystem I subunit chloroplast precursor & $8.20 E-90$ & 20 & 2 & 11 & & & 3 \\
\hline 1313.7 & Photosystem I light-harvesting protein & $5.20 E-133$ & 28 & 4 & 18 & & & 3 \\
\hline 1313.76 & Photosystem I light-harvesting protein & $1.80 \mathrm{E}-130$ & 23 & 2 & 11 & & & 1 \\
\hline 1504.25 & Chlorophyll a/b binding & $2.30 E-158$ & 31 & 2 & 6 & & & 1 \\
\hline 1611.13 & Photosystem I subunit XI precursor & $5.20 E-98$ & 46 & 2 & 7 & & & 11 \\
\hline 1615.55 & Porphobilinogen deaminase & $0.00 \mathrm{E}+00$ & 38 & & & & & 5 \\
\hline 1759.19 & Photosystem I light-harvesting protein & $1.10 E-126$ & 79 & 2 & 2 & & & 5 \\
\hline 2265.19 & Photosystem II oxygen-evolving complex & 2.10E-81 & 26 & 2 & 10 & & & 0 \\
\hline 2779.8 & Light-harvesting chlorophyll a/b-binding protein & $1.20 E-155$ & 27 & 4 & 19 & 3 & 15 & 1 \\
\hline \multicolumn{9}{|c|}{ Calvin-Benson cycle/carbon capture and concentration } \\
\hline 101.72 & Phosphoglycerate kinase & $0.00 \mathrm{E}+00$ & 49 & 3 & 8 & & & 3 \\
\hline 669.32 & Glyceraldehyde-3-phosphate dehydrogenase & $0.00 \mathrm{E}+00$ & 43 & 8 & 24 & & & 4 \\
\hline 971.4 & Low- $\mathrm{CO}_{2}$ inducible protein & $0.00 \mathrm{E}+00$ & 55 & 6 & 22 & 3 & 12 & 10 \\
\hline 1137.114 & Transketolase & $0.00 \mathrm{E}+00$ & 77 & 3 & 5 & & & 9 \\
\hline 1250.71 & Glyceraldehyde-3-phosphate dehydrogenase type I & $0.00 \mathrm{E}+00$ & 38 & 2 & 9 & 2 & 9 & 5 \\
\hline 1605.9 & Glyceraldehyde-3-phosphate dehydrogenase type I & $8.60 E-125$ & 22 & 5 & 33 & 2 & 12 & 2 \\
\hline 1655.2 & Ribulose bisphosphate small subunit precursor & $7.60 E-84$ & 15 & 5 & 44 & 2 & 20 & 4 \\
\hline 1691.8 & Fructose bisphosphate aldolase & $0.00 \mathrm{E}+00$ & 41 & 6 & 18 & & & 4 \\
\hline \multicolumn{9}{|c|}{ Protein synthesis } \\
\hline 352.92 & Glutamine synthetase & $0.00 \mathrm{E}+00$ & 42 & 2 & 8 & & & 10 \\
\hline 355.1 & Elongation factor tu & $0.00 \mathrm{E}+00$ & 45 & 3 & 10 & & & 2 \\
\hline 407.59 & Choline dehydrogenase & $0.00 E+00$ & 64 & 2 & 4 & & & 7 \\
\hline 564.107 & $60 S$ ribosomal protein & 4.30E-107 & 17 & 2 & 17 & & & 3 \\
\hline 759.8 & Eukaryotic translation elongation factor 1 alpha 2 & $0.00 E+00$ & 49 & 16 & 39 & 7 & 17 & 10 \\
\hline 835.5 & Carbamoyl-phosphate synthase & $0.00 \mathrm{E}+00$ & 117 & 2 & 2 & & & 17 \\
\hline 1136.46 & Elongation factor 2 & $0.00 \mathrm{E}+00$ & 68 & 2 & 4 & & & 16 \\
\hline 1412.67 & Homocysteine S-methyltransferase & $0.00 \mathrm{E}+00$ & 109 & 2 & 4 & & & 20 \\
\hline 2123.11 & Glutamate-1-semialdehyde-aminomutase & $0.00 \mathrm{E}+00$ & 47 & 3 & 10 & & & 7 \\
\hline \multicolumn{9}{|c|}{ TCA cycle/energy generation } \\
\hline 291.19 & ATP synthase CF1-beta subunit & $0.00 \mathrm{E}+00$ & 49 & 5 & 18 & & & 3 \\
\hline 291.6 & ATP synthase CF1 alpha subunit & $0.00 \mathrm{E}+00$ & 55 & 4 & 11 & & & 1 \\
\hline 1889.22 & Dihydrolipoyl (dihydrolipamide) dehydrogenase & $0.00 \mathrm{E}+00$ & 53 & 2 & 5 & & & 6 \\
\hline \multicolumn{9}{|c|}{ Stress response/signaling cascades } \\
\hline 545.25 & L-Ascorbate peroxidase & $1.20 E-153$ & 43 & 2 & 8 & & & 9 \\
\hline 1123.1 & Calcium calmodulin-dependent protein kinase & $3.50 E-100$ & 25 & 2 & 13 & & & 1 \\
\hline 1196.84 & Hop-interacting protein thi045 & $1.80 E-110$ & 25 & 8 & 45 & 3 & 18 & 2 \\
\hline \multicolumn{9}{|c|}{ Fatty acid biosynthesis } \\
\hline 2978.6 & Biotin carboxylase & $2.40 E-164$ & 31 & 2 & 10 & & & 2 \\
\hline \multicolumn{9}{|c|}{ Cell maintenance and growth } \\
\hline 439.32 & Beta-tubulin & $0.00 \mathrm{E}+00$ & 47 & 3 & 8 & & & 13 \\
\hline
\end{tabular}

its three isozymes are differentially regulated, one is downregulated, while the other two are upregulated during nitrogen stress (Guarnieri et al., 2013). Interestingly, S-nitrosylation of GAPDH has been shown to inhibit this enzyme and cause a shift in its activity where it localizes to the nucleus and acts as an $S$-nitrosylase and $S$-nitrosylates other target proteins (Astier and Lindermayr, 2012; Zaffagnini et al., 2013). Thus, $S$-nitrosylated GAPDH could mediate global transcriptional 
and metabolic regulatory alterations in C. vulgaris. We also identified FBA as $S$-nitrosylated under nitrogen-replete growth. In Arabidopsis, FBA is important for modulating stress responses and is inhibited by S-nitrosylation (van der Linde et al., 2011; Lu et al., 2012). Together with the inhibition of GAPDH, FBA inhibition could result in increased carbon flux through the oxidative branch of the pentose phosphate pathway under nitrogen-replete growth.

These data support endogenously produced NO during nitrate metabolism as a potential signaling molecule and indicate that protein S-nitrosylation could be a common post-translational modification utilized by $C$. vulgaris to regulate responses to nitrogen availability. Interestingly, a significant percentage (30\%) of the $S$-nitrosylated proteins are differentially regulated under nitrogen-replete and nitrogen-deplete conditions (Guarnieri et al., 2013), suggesting that NO might be a key post-translational regulator of lipid biosynthesis in C. vulgaris. Collectively, the data presented here provide insight into the physiological role of S-nitrosylation as it relates to lipid accumulation, which can inform C. vulgaris engineering strategies to enable algae-based biofuels.

\section{MATERIALS AND METHODS}

\section{Culture Conditions and Biotin Switch}

Chlorella vulgaris UTEX395 was grown in BBM supplemented with $3 \mathrm{mM} \mathrm{NaNO}$ to an $\mathrm{OD}_{750 \mathrm{~nm}}$ of 2 as previously described (Guarnieri et al., 2011). Equal concentrations of logarithmically growing cells were harvested, washed in nitrate-free BBM, suspended in $\mathrm{BBM}$ with or without $3 \mathrm{mM} \mathrm{NaNO}_{3}$ to $\mathrm{OD}_{750 \mathrm{~nm}}=2.0$, and grown in $500 \mathrm{~mL}$ shake flasks. After $24 \mathrm{~h}$ of incubation, whole cell lysates were prepared and enriched for $S$-nitrosylated proteins using the biotin switch pull-down assay as previously described (Jaffrey and Snyder, 2001; Forrester et al., 2009).

\section{Fluorometric Detection of NO}

Chlorella vulgaris grown in $\mathrm{BBM}$ with $3 \mathrm{mM} \mathrm{NaNO}_{3}$ to an $\mathrm{OD}_{750 \mathrm{~mm}}$ of 2 were pelleted, washed $2 \times$ in nitrate-free BMM, and resuspended at a concentration of $1 \times 10^{6}$ cells $/ \mathrm{mL}$ in BBM pH 4.5 supplemented with $1 \mathrm{mM} \mathrm{NaNO}$ or $\mathrm{NaNO}_{3}$ for $1 \mathrm{~h}$. After treatment, equal concentrations of $\mathrm{NaNO}_{2}$-treated, $\mathrm{NaNO}_{3}$-treated, and untreated cells were stained with 4,5-diaminofluorescein (Cayman Chemical, Ann Arbor, MI, USA) to detect nitric oxide production fluorometrically.

\section{Mass Spectrometry Analysis}

Peptides were purified and concentrated using an online enrichment column (Thermo Scientific $5 \mu \mathrm{m}, 100 \mu \mathrm{m}$ ID $\times 2 \mathrm{~cm} \mathrm{C18}$ column). Subsequent chromatographic separation was performed on a reverse phase nanospray column (Thermo Scientific EASYnano-LC, $3 \mu \mathrm{m}, 75 \mu \mathrm{m}$ ID $\times 100 \mathrm{~mm}$ C18 column) using a 30 min linear gradient from $10-30 \%$ buffer B (100\% ACN, $0.1 \%$ formic acid) at a flow rate of $400 \mathrm{~nL} / \mathrm{min}$. Peptides were eluted directly into the mass spectrometer (Thermo Scientific
Orbitrap Velos) and spectra were collected over a $\mathrm{m} / \mathrm{z}$ range of 400-2000 Da using a dynamic exclusion limit of two MS/MS spectra of a given peptide mass for $30 \mathrm{~s}$ (exclusion duration of $90 \mathrm{~s}$ ). The instrument was operated in Orbitrap-LTQ mode where precursor measurements were acquired in the orbitrap $(60,000$ resolution) and MS/MS spectra (top 20) were acquired in the LTQ ion trap with a normalized collision energy of $35 \mathrm{kV}$. Compound lists of the resulting spectra were generated using Xcalibur 2.2 software (Thermo Scientific) with an $S / N$ threshold of 1.5 and 1 scan/group.

\section{Protein Identification}

Tandem mass spectra were extracted, charge state deconvoluted and deisotoped by ProteoWizard (MS-Convert) version 3.0. All MS/MS samples were analyzed using Mascot (Matrix Science, London, UK; version 2.3.02). Mascot was set up to search the Cv395_Maker_7100_peps database (unknown version, 7,100 entries) assuming the digestion enzyme trypsin. Mascot was searched with a fragment ion mass tolerance of $0.80 \mathrm{Da}$ and a parent ion tolerance of $20 \mathrm{ppm}$. Oxidation of methionine and carbamidomethyl of cysteine were specified in Mascot as variable modifications. Scaffold (version Scaffold_4.3.2, Proteome Software Inc., Portland, OR, USA) was used to validate MS/ MS-based peptide and protein identifications. Peptide identifications were accepted if they could be established at greater than 85.0\% probability by the Peptide Prophet algorithm (Keller et al., 2002) with Scaffold delta-mass correction. Protein identifications were accepted if they could be established at greater than $80.0 \%$ probability and contained at least one identified peptide. Protein probabilities were assigned by the Protein Prophet algorithm (Nesvizhskii et al., 2003). Proteins that contained similar peptides and could not be differentiated based on MS/MS analysis alone were grouped to satisfy the principles of parsimony. All identified peptides had a $>95 \%$ probability of correct identification based on a BLASTP search of the predicted peptides against the predicted proteins present in the draft C. vulgaris genome (Accession\#: LDKB01000000). S-nitrosylated targets identified here under nitrogen-deplete and -replete conditions are presented in Table $\mathbf{1}$ and deposited as an excel file at Figshare at the following link: https://figshare.com/s/39ea5540257b0efa9d5d.

\section{Quality Control}

Instrument functionality and stability was monitored using the MassQC software (Proteome Software). This software uses a set of quantitative metrics developed by the National Institute of Science and Technology in collaboration with the National Cancer Institute's Clinical Proteomic Technologies for Cancer (CPTC) that monitor technical variability in mass spectrometrybased proteomics instrumentation. Quality control samples containing a mixture of six trypsin digested bovine proteins were injected at least once every $24 \mathrm{~h}$ throughout the analysis, and the data from this run were analyzed using the MassQC software. Values for all metrics were within normal limits throughout the duration of the experiment indicating instrument stability and data robustness. 


\section{AUTHOR CONTRIBUTIONS}

$\mathrm{CH}$ and EK designed experimental strategies. $\mathrm{CH}$ performed experiments and $\mathrm{CH}, \mathrm{MG}$, and $\mathrm{EK}$ analyzed data. $\mathrm{CH}, \mathrm{MG}$, and EK wrote the manuscript.

\section{ACKNOWLEDGMENTS}

The authors would like to thank the Colorado State University Proteomics and Metabolomics Facility for assistance with

\section{REFERENCES}

Astier, J., and Lindermayr, C. (2012). Nitric oxide-dependent posttranslational modification in plants: an update. Int. J. Mol. Sci. 13, 15193-15208. doi:10.3390/ ijms131115193

Converti, A., Casazza, A. A., Ortiz, E. Y., Perego, P., and Del Borghi, M. (2009). Effect of temperature and nitrogen concentration on the growth and lipid content of Nannochloropsis oculata and Chlorella vulgaris for biodiesel production. Chem. Eng. Process. 48, 1146-1151. doi:10.1016/j.cep.2009.03.006

Day, J. G., Slocombe, S. P., and Stanley, M. S. (2012). Overcoming biological constraints to enable the exploitation of microalgae for biofuels. Bioresour. Technol. 109, 245-251. doi:10.1016/j.biortech.2011.05.033

Forrester, M. T., Foster, M. W., Benhar, M., and Stamler, J. S. (2009). Detection of protein S-nitrosylation with the biotin-switch technique. Free Radic. Biol. Med. 46, 119-126. doi:10.1016/j.freeradbiomed.2008.09.034

Guarnieri, M. T., Nag, A., Smolinski, S. L., Darzins, A., Seibert, M., and Pienkos, P. T. (2011). Examination of triacylglycerol biosynthetic pathways via de novo transcriptomic and proteomic analyses in an unsequenced microalga. PLoS ONE 6:e25851. doi:10.1371/journal.pone.0025851

Guarnieri, M. T., Nag, A., Yang, S., and Pienkos, P. T. (2013). Proteomic analysis of Chlorella vulgaris: potential targets for enhanced lipid accumulation. J. Proteomics 93, 245-253. doi:10.1016/j.jprot.2013.05.025

Henard, C. A., Tapscott, T., Crawford, M. A., Husain, M., Doulias, P.-T., Porwollik, S., et al. (2014). The 4-cysteine zinc-finger motif of the RNA polymerase regulator DksA serves as a thiol switch for sensing oxidative and nitrosative stress. Mol. Microbiol. 91, 790-804. doi:10.1111/mmi.12498

Hu, Q., Sommerfeld, M., Jarvis, E., Ghirardi, M., Posewitz, M., Seibert, M., et al. (2008). Microalgal triacylglycerols as feedstocks for biofuel production: perspectives and advances. Plant J. 54, 621-639. doi:10.1111/j.1365-313X.2008.03492.x

Ikaran, Z., Suárez-Alvarez, S., Urreta, I., and Castañón, S. (2015). The effect of nitrogen limitation on the physiology and metabolism of Chlorella vulgaris var L3. Algal Res. 10, 134-144. doi:10.1016/j.algal.2015.04.023

Jaffrey, S. R., and Snyder, S. H. (2001). The biotin switch method for the detection of S-nitrosylated proteins. Sci. STKE 2001, pl1. doi:10.1126/stke.2001.86.pl1

Keller, A., Nesvizhskii, A. I., Kolker, E., and Aebersold, R. (2002). Empirical statistical model to estimate the accuracy of peptide identifications made by MS/ MS and database search. Anal. Chem. 74, 5383-5392. doi:10.1021/ac025747h

Liang, Y., Sarkany, N., and Cui, Y. (2009). Biomass and lipid productivities of Chlorella vulgaris under autotrophic, heterotrophic and mixotrophic growth conditions. Biotechnol. Lett. 31, 1043-1049. doi:10.1007/s10529-009-9975-7

Lu, W., Tang, X., Huo, Y., Xu, R., Qi, S., Huang, J., et al. (2012). Identification and characterization of fructose 1,6-bisphosphate aldolase genes in Arabidopsis reveal a gene family with diverse responses to abiotic stresses. Gene 503, 65-74. doi:10.1016/j.gene.2012.04.042

Maldonado-Alconada, A. M., Echevarría-Zomeño, S., Lindermayr, C., RedondoLópez, I., Durner, J., and Jorrín-Novo, J. V. (2011). Proteomic analysis of Arabidopsis protein S-nitrosylation in response to inoculation with Pseudomonas syringae. Acta Physiol. Plant. 33, 1493-1514. doi:10.1007/s11738-010-0688-2

Mu, J., Li, S., Chen, D., Xu, H., Han, F., Feng, B., et al. (2015). Enhanced biomass and oil production from sugarcane bagasse hydrolysate (SBH) by heterotrophic oleaginous microalga Chlorella protothecoides. Bioresour. Technol. 185, 99-105. doi:10.1016/j.biortech.2015.02.082 the mass spectrometry analysis and subsequent protein identification.

\section{FUNDING}

This work was funded by the Laboratory Directed Research and Development (LDRD) program of the National Renewable Energy Laboratory (NREL). This work was also supported in part by the Department of Energy, Office of Energy Efficiency and Renewable Energy (EERE) under Agreement No. 28812.

Mur, L. A. J., Mandon, J., Persijn, S., Cristescu, S. M., Moshkov, I. E., Novikova, G. V., et al. (2013). Nitric oxide in plants: an assessment of the current state of knowledge. AoB Plants 5, pls052. doi:10.1093/aobpla/pls052

Nesvizhskii, A. I., Keller, A., Kolker, E., and Aebersold, R. (2003). A statistical model for identifying proteins by tandem mass spectrometry. Anal. Chem. 75, 4646-4658. doi:10.1021/ac0341261

Ortega-Galisteo, A. P., Rodríguez-Serrano, M., Pazmiño, D. M., Gupta, D. K., Sandalio, L. M., and Romero-Puertas, M. C. (2012). S-Nitrosylated proteins in pea (Pisum sativum L.) leaf peroxisomes: changes under abiotic stress. J. Exp. Bot. 63, 2089-2103. doi:10.1093/jxb/err414

Puyaubert, J., Fares, A., Rézé, N., Peltier, J.-B., and Baudouin, E. (2014). Identification of endogenously S-nitrosylated proteins in Arabidopsis plantlets: effect of cold stress on cysteine nitrosylation level. Plant Sci. 21, 150-156. doi:10.1016/ j.plantsci.2013.10.014

Quinn, J. C., and Davis, R. (2015). The potentials and challenges of algae based biofuels: a review of the techno-economic, life cycle, and resource assessment modeling. Bioresour. Technol. 184, 444-452. doi:10.1016/j.biortech.2014.10.075

Sakihama, Y., Nakamura, S., and Yamasaki, H. (2002). Nitric oxide production mediated by nitrate reductase in the green alga Chlamydomonas reinhardtii: an alternative NO production pathway in photosynthetic organisms. Plant Cell Physiol. 43, 290-297. doi:10.1093/pcp/pcf034

Samuel, M., Mirko, Z., Xing-Huang, G., Lemaire Stéphane, D., and Marchand Christophe, H. (2014). Insight into protein S-nitrosylation in Chlamydomonas reinhardtii. Antioxid. Redox Signal. 21, 1271-1284. doi:10.1089/ars.2013.5632

van der Linde, K., Gutsche, N., Leffers, H.-M., Lindermayr, C., Müller, B., Holtgrefe, S., et al. (2011). Regulation of plant cytosolic aldolase functions by redox-modifications. Plant Physiol. Biochem. 49, 946-957. doi:10.1016/j.plaphy.2011.06.009

Vanzo, E., Ghirardo, A., Merl-Pham, J., Lindermayr, C., Heller, W., Hauck, S. M., et al. (2014). S-nitroso-proteome in poplar leaves in response to acute ozone stress. PLoS ONE 9:e106886. doi:10.1371/journal.pone.0106886

Williams, P. J. B., and Laurens, L. M. L. (2010). Microalgae as biodiesel \& biomass feedstocks: review \& analysis of the biochemistry, energetics \& economics. Energy Environ. Sci. 3, 554-590. doi:10.1039/b924978h

Zaffagnini, M., De Mia, M., Morisse, S., Di Giacinto, N., Marchand, C. H., Maes, A., et al. (2016). Protein S-nitrosylation in photosynthetic organisms: a comprehensive overview with future perspectives. Biochim. Biophys. Acta 1864, 952-966. doi:10.1016/j.bbapap.2016.02.006

Zaffagnini, M., Morisse, S., Bedhomme, M., Marchand, C. H., Festa, M., Rouhier, N., et al. (2013). Mechanisms of nitrosylation and denitrosylation of cytoplasmic glyceraldehyde-3-phosphate dehydrogenase from Arabidopsis thaliana. J. Biol. Chem. 288, 22777-22789. doi:10.1074/jbc.M113.475467

Conflict of Interest Statement: The authors declare that the research was conducted in the absence of any commercial or financial relationships that could be construed as a potential conflict of interest.

Copyright (C) 2017 Henard, Guarnieri and Knoshaug. This is an open-access article distributed under the terms of the Creative Commons Attribution License (CC BY). The use, distribution or reproduction in other forums is permitted, provided the original author(s) or licensor are credited and that the original publication in this journal is cited, in accordance with accepted academic practice. No use, distribution or reproduction is permitted which does not comply with these terms. 